\title{
Does complete deficiency of muscle $\alpha$ actinin 3 alter functional capacity in elderly women? A preliminary report
} A F San Juan, F Gomez-Gallego, S Cañete, C Santiago, M Pérez, A Lucia

The sarcomeric protein $\alpha$ actinin 3 is localised to the $Z$ line of fast fibres, which are responsible for generating forceful muscle contractions at high velocity. However, a substantial proportion of healthy humans are totally deficient in this protein as they are homozygous for a premature stop codon polymorphism (R577X) in the ACTN3 gene. The purpose of this preliminary study was to assess if the presence or absence of $\alpha$ actinin 3 influences the deleterious effects of ageing on muscle output and functional capacity.

$\mathrm{T}$ he $\alpha$ actinins are an ancient family of actin binding proteins that play both structural and contractile roles in muscle contraction in several species. ${ }^{1}$ The two sarcomeric $\alpha$ actinin isoforms in humans, $\alpha$ actinin 2 and $\alpha$ actinin 3 , constitute the predominant component of the sarcomeric $\mathrm{Z}$ line, where they form a lattice structure that anchors together actinin-containing thin filaments and stabilises the muscle contractile apparatus. $^{2}$ Besides their mechanical role, both sarcomeric $\alpha$ actinins interact with proteins involved in numerous signalling and metabolic pathways. ${ }^{13}$ The pattern of expression of the two isoforms has diverged through mammalian evolution, with $\alpha$ actinin 2 becoming predominantly expressed in type I, fatigue resistant (oxidative) fibres and cardiomyoctes, whereas the expression of $\alpha$ actinin 3 is largely restricted to the fast glycolytic type II fibres ${ }^{13}$ which are responsible for generating forceful contractions at high velocity.

Despite the evolutionary conservation of $\alpha$ actinin 3, a substantial proportion of healthy humans are totally deficient in this protein as they are homozygous for a premature stop codon polymorphism (R577X) in the gene (ACTN3) encoding $\alpha$ actinin $3 .{ }^{4}$ This genetic variant is associated with no disease phenotype, although a recent study suggests that the presence of $\alpha$ actinin 3 confers some benefit on elite sprint/ power athletes, who are required to generate very rapid, forceful contractions in their sport events; this effect was more pronounced in female athletes. ${ }^{5}$ Conversely, the absence of $\alpha$ actinin 3 (associated with 577XX) appears to be more common in endurance athletes, although the results reached statistical significance only in female athletes. ${ }^{5}$

No previous investigation has specifically studied the possible effects of the ACTN3 genotypes on the functional capacity of the elderly. Ageing affects functional capacity through several changes at the peripheral muscle level that impair the capacity of muscle to produce energy and generate forceful contractions. These include denervation muscle atrophy, ${ }^{6-9}$ particularly affecting type II fibres ${ }^{7}$ and a decline in total creatine and phosphocreatine concentration in muscle. ${ }^{11}$ The age decline in physical function and the ability to produce muscle power appears to be greater in women than in men, at least for large muscle exercise. ${ }^{12}$ Considering all the above age associated phenomena that mainly result in a decreased potential for muscle force generation, particularly in women, together with the fact that both longevity and the proportion of elderly people engaging in fitness programmes are increasing in Western societies, it would be worth while to explore the effect of the ACTN3 genotype on ageing muscle. We therefore aimed to compare several indices of functional capacity in elderly women with reference to their ACTN3 genotype.

\section{METHODS}

The institutional research ethics committee (Universidad Europea de Madrid, Spain) approved the study. After giving their written informed consent, 23 healthy, non-athletic elderly women (age 61-80 years) volunteered for this investigation. A previous medical examination (including resting electrocardiogram) was used to ensure that all the subjects were free of disease. None was taking any drug that could significantly alter physiological responses to exercisefor example, $\beta$ blockers. Participants had a moderately active lifestyle, which was defined by them as walking (at a comfortable-moderate pace) for 30-60 $\mathrm{min} /$ day, 4-5 days/ week but not performing strenuous exercise such as running, swimming, or cycling. Before the tests, physical activity energy expenditure was determined using a Caltrac uniaxial accelerometer (Muscle Dynamics Fitness Network, Torrance, California, USA).

All participants performed the same battery of tests, consisting of a blood analysis followed by anthropometric measurements and graded exercise test (lst day), bench press test (2nd day), and functional tests (3rd day: lower body functional test and an outdoor one mile walk test). During the two days before testing, subjects were instructed to refrain from performing strenuous physical activity.

Blood samples were obtained from an antecubital vein to obtain haemograms and to extract genomic DNA according to standard phenol/chloroform procedures followed by alcohol precipitation. The polymerase chain reaction was performed to amplify the sequence containing the mutation. ACTN3 genotypes were established by enzymatic digestion of amplicons with $D d e$ I. $^{3}$ The R577X change creates a restriction site resulting in fragments of 108, 97 and $86 \mathrm{bp}$. Digestion of the R577 allele resulted in fragments of 205 and $86 \mathrm{bp}$. Digestion products were analyzed by capillary electrophoresis in an ABI Prism 310 genetic analyser.

Body fat percentage was assessed indirectly from subcutaneous skinfolds at four sites (triceps, biceps, subscapular, and iliac crest). ${ }^{13}$

All the graded tests (workload increases of $15 \mathrm{~W} / \mathrm{min}$ starting at $15 \mathrm{~W}$ ) were performed on a cycle ergometer until volitional exhaustion. Gas exchange data were collected continuously using an automated breath by breath system (Vmax 29C; Sensormedics, Yorba Linda, California, USA) to determine peak oxygen uptake $\left(\dot{\mathrm{V}}_{2} \mathrm{PEAK}\right.$, recorded as the highest $\dot{\mathrm{V}}_{2}$ value obtained for any one minute period), peak power output, ${ }^{14}$ and the ventilatory threshold. ${ }^{15}$ 
Table 1 Main characteristics of subjects with complete deficiency of $\alpha$ actinin (XX homozygotes) or with the other two genotypes (RX and RR) for the ACTN3 gene

\begin{tabular}{lll}
\hline & $\begin{array}{l}\text { XX } \\
\text { (n=5) }\end{array}$ & $\begin{array}{l}\text { RX/RR } \\
\text { (n= 18) }\end{array}$ \\
\hline Age (years) & $67(5)$ & $67(6)$ \\
Height (cm) & $158(7)$ & $156(7)$ \\
Weight (kg) & $68(7)$ & $63(10)$ \\
$\%$ body fat & $18.7(2.0)$ & $18.9(2.8)$ \\
Estimated daily physical activity & $560(36)$ & $542(48)$ \\
(kcal/day) & & \\
Packed cell volume (\%) & $43.3(1.4)$ & $41.8(0.8)$ \\
Haemoglobin (g/dl) & $14.5(0.7)$ & $13.9(0.3)$ \\
$\dot{V} o_{2}$ PEAK (ml/kg/min) & $18.4(2.0)$ & $20.7(1.3)$ \\
PPO (W/kg) & $1.5(0.2)$ & $1.7(0.1)$ \\
$\dot{V} o_{2}$ (ml/kg/min) at VT & $11.5(1.1)$ & $12.0(0.8)$ \\
One mile walk test (s) & $980(43)$ & $932(18)$ \\
Sit-stand test (s) & $9.1(0.3)$ & $9.5(0.3)$ \\
1RM bench press (kg) & $23.9(2.8)$ & $22.7(1.4)$ \\
\hline
\end{tabular}

Values are mean (SEM). There are no significant differences between the means $(p>0.05)$.

$\dot{V}_{2}$ PEAK, Peak oxygen uptake; PPO, peak power output; VT, ventilatory threshold; 1RM, one repetition maximum.

Subjects performed a one repetition maximum test on the bench press apparatus (Technogym, Gambettola, Italy) to determine their maximal upper body dynamic strength. ${ }^{16}$ Functional endurance was assessed with a one mile walk test performed on flat terrain. Lower extremity functional performance was also determined using a sit-stand test. ${ }^{17}$ In an effort to eliminate anticipated learning effects and establish reliability of both sit-stand and strength tests, ${ }^{16}$ before the start of the study the subjects performed three to four familiarisation sessions with both types of test followed by two tests for each exercise task (separated by one week), which showed an intraclass correlation coefficient of $\geqslant 0.95$.

\section{Statistical analysis}

The Mann-Whitney U test was used to compare average daily physical activity energy expenditure, blood and physiological variables, and functional capacity between subjects with total deficiency of $\alpha$ actinin 3 (XX homozygotes) and both RX heterozygotes and RR homozygotes. The level of significance was set at 0.05 .

\section{RESULTS AND DISCUSSION}

The proportion of XX homozygotes was $\sim 22 \%$ (five out of 23 subjects). Table 1 shows their main characteristics and the results of exercise and functional tests. No significant difference was found between XX homozygotes and the rest of the subjects.

In this study, we examined for the first time whether complete deficiency of $\alpha$ actinin 3 affects the functional capacity of elderly women (mean age $\sim 70$ years). Two variables that can affect exercise performance irrespective of genetic variations (blood oxygen transport capacity and level of physical activity) were controlled for. Our results suggest that, in this population group, complete deficiency of this muscle protein does not affect widely accepted indices of health status, longevity, and aerobic fitness such as ventilatory threshold ${ }^{18}$ and peak oxygen uptake, ${ }^{19}$ performance during functional tests (sit-stand test, one mile walk test), or maximal muscle strength. Of especial practical applicability was the lack of impairment in the functional tests, particularly the sit-stand test, in patients with complete $\alpha$ actinin 3 deficiency (577XX). A decrease in the functional ability to perform tasks of daily living has been associated with an increase in mortality. ${ }^{2021}$ Several factors that are affected by ageing, such as balance, coordination,

\section{What is already known on this topic}

- The sarcomeric protein $\alpha$ actinin 3 plays a crucial role in generating forceful skeletal muscle contractions at high velocity

\section{What this study adds}

- Although more research is necessary using larger population groups, our preliminary results show that complete deficiency of $\alpha$ actinin 3 does not affect maximum aerobic capacity, functional capacity, or maximal muscle strength in elderly women

submaximal strength, and the ability to rapidly generate muscle torque, are important performance determinants in the sit-stand test. ${ }^{17}$ These factors, which are important for preventing falls during daily physical tasks involving lower body muscle power such as sitting and rising from a chair or walking downstairs, seem to be unaffected by complete $\alpha$ actinin 3 deficiency.

Although more research is necessary using larger population groups, our preliminary finding is relevant and of practical applicability, as the longevity of Western societies has significantly increased over the last few decades. It is therefore necessary to confirm that common genetic variations do not aggravate the negative effects that ageing per se has on physical function.

\section{ACKNOWLEDGEMENTS}

We are indebted to Professor Kathryn North for her comments.

\section{Authors' affiliations}

A F S Juan, F Gomez-Gallego, S Cañete, C Santiago, M Pérez, A Lucia, Universidad Europea de Madrid, Spain

Competing interests: none declared

Correspondence to: Professor Lucia, Universidad Europea de Madrid, Madrid 28670, Spain; alejandro.lucia@uem.es

Accepted 11 April 2005

\section{REFERENCES}

1 MacArthur DG, North KN. A gene for speed? The evolution and function of $\alpha$ actinin 3. BioEssays 2004;26:786-95.

2 Squire JM. Architecture and function in the muscle sarcomere. Curr Opin Struct Biol 1997;7:247-57.

3 Mills M, Tang R, Weinberger R, et al. Differential expression of the actinbinding proteins, $\alpha$-actinin- 2 and -3 , in different species: implications for the evolution of functional redundancy. Hum Mol Gen 2001;10:1335-46.

4 North KN, Yang N, Wattanasirichaigoon D, et al. A common nonsense mutation results in $\alpha$ actinin 3 deficiency in the general population. Nat Genet 1999;21:353-4.

5 Yang N, MacArthur DG, Gulbin JP, et al. ACTN3 genotype is associated with human elite athletic performance. Am J Hum Genet 2003;73:627-31.

6 Cartee GD. Aging skeletal muscle: response to exercise. Exerc Sport Sci Rev 1994;22:91-20.

7 Doherty TJ, Vandervoort AA, Brown WF. Effects of ageing on the motor unit: a brief review. Can J Appl Physiol 1993;18:331-58.

8 Doherty TJ, Vandervoort AA, Taylor AW, et al. Effects of motor unit losses on strength in older men and women. J Appl Physiol 1993;74:868-74.

9 Grabiner MD, Enoka RA. Changes in movement capabilities with aging. Exerc Sport Sci Rev 1995;23:65-104.

10 Engel WK. Selective and nonselective susceptibility of muscle fiber types: a new approach to human neuromuscular diseases. Arch Neurol 1970;22:97-117.

11 Tarnopolski MA. Potential benefits of creatine monohydrate supplementation in the elderly. Curr Opin Clin Nutr Metab Care 2000;3:497-502. 
12 Tanaka H, Seals DR. invited review: dynamic exercise performance in Masters athletes: insight into the effects of primary human aging on physiological functional capacity. J Appl Physiol 2003;95:2152-62.

13 Durnin JV, Womersley J. Body fat assessed from total body density and its estimation from skinfold: measurements on 481 men and women aged from 16 to 72 years. Br J Nutr 1974;32:77-97.

14 Kuipers H, Verstappen FT, Keizer HA, et al. Variability of aerobic performance in the laboratory and its physiologic correlates. Int J Sports Med 1985:6:197-201

15 Wasserman K, Mcllroy MB. Detecting the threshold for anaerobic metabolism in cardiac patients during exercise. Am J Cardiol 1964; 14:844-52.

16 Gotshalk LA, Volek JS, Staron RS, et al. Creatine supplementation improves muscular performance in older men. Med Sci Sports Exerc 2002;34:537-43.
17 Guralnik JM, Simonsick EM, Ferrucci L, et al A short physical performance battery assessing lower extremity function: association with self-reported disability and prediction of mortality and nursing home admission. J Gerontol 1994:49:M85-94.

18 Meyer T, Lucia A Earnest CP et al A conceptual framework for performance diagnosis and training prescription from submaximal parameters: theory and application. Int J Sports Med 2004; (suppl 1):S38-48.

19 Myers J, Prakash M, Froelicher V, et al. Exercise capacity and mortality among men referred for exercise testing. N Engl J Med 2002:4:793-801.

20 Beltran B, Cuadrado C, Martin LM, et al. Activities of daily living in the Spanish elderly. Association with mortality. J Nutr Health Aging 2001;5:259-260.

21 Kushiro W, Yokoyama T, Date C, et al. Association of activities of daily living and indices of mental status with subsequent 20-year all-cause mortality in an elderly Japanese population. Nurs Health Sci 2002;4(suppl 3):A5 